\title{
Chapter 1 Scaling of the aeroacoustics of high-speed trains
}

Andreas Lauterbach, Sigfried Loose, and Klaus Ehrenfried

\section{Summary}

The present study focuses on the scaling of aeroacoustic sound sources of a highspeed train. To cover a wide Reynolds number range the experimental investigations are carried out with a 1:25 Inter City Express 3 model by measuring in two wind tunnels by means of microphone array technique. The facilities are the Aeroacoustic Wind tunnel (AWB) of the German Aerospace Center (DLR) in Brunswick, which provides nearly perfect acoustical conditions, and in the Cryogenic wind tunnel (DNW-KKK) of the DNW (German - Dutch wind tunnels) in Cologne, which allows measurements at higher Reynolds numbers. Two sources of sound with different characteristics are identified at Reynolds numbers up to $R e=0.46$ mio. The aeroacoustic noise from the bogie section is dominant for frequencies $f<5 \mathrm{kHz}$ and can be characterised by cavity mode excitation. The pantograph is the dominant source of sound above $f=5 \mathrm{kHz}$ with a Aeolian tone characteristic. Additionally aeroacoustic measurements at higher Reynolds numbers of up to 3.70 mio have been conducted in the DNW-KKK. By cooling down one can increase the Reynolds number, and besides, this wind tunnel admits to vary the Mach and Reynolds numbers independently. Drawback of this facility is that it is not optimised for aeroacoustic experiments and reflexions as well as the high background noise level can disturb the results. These measurements revealed only a weak Reynolds number dependence of the noise source generated at the first bogie.

German Aerospace Center (DLR), Institute of Aerodynamics and Flow Technology dept. Fluid Systems

Bunsenstrasse 10

37073 Goettingen

Germany

Tel.: +49-(0)551-7092464

Fax: +49-(0)551-7092241

e-mail: andreas.lauterbach@dlr.de 


\subsection{Introduction}

Modern high-speed trains allow operational speeds up to $350 \mathrm{~km} / \mathrm{h}$ and for this range of velocities aerodynamic noise exceeds all other sources of sound. For reducing the aerodynamic noise a detailed knowledge of the distribution and the properties of the sound sources is necessary. In the field of acoustics of high-speed trains both, investigations on the full-scale vehicles as well as testings in wind tunnels on models have been done. Full-scale tests can be conducted under real conditions and appropriate Reynolds numbers. The disadvantage is that these testings only can be performed when the train is already in operation, and so it is not possible to analyse acoustics during the design process. Most of the earlier publications report on full-scale testings and have their focus on the so-called wheel-rail noise (see Barsikow et al. [3]). At the same time modern microphone array techniques became an effective tool for the localisation of aeroacoustic sound sources (see Martens et al. [13]). For the prediction of the aeroacoustic properties during the design process Yamazaki et al. [17] investigated aerodynamic noise of a simplified down scaled train model at Reynolds numbers up to 2 mio in the wind tunnel, using beamforming techniques. This qualitative study was focused on noise sources generated by the bogie cavities and the gaps between the coaches. Based on this measurements noise reduction measures were applied to a full scale train. Other wind tunnel studies just point out investigations of parts of trains, e.g. the pantograph (see [14, 8]). Still an open issue is a comprehensive quantitative aeroacoustic study of trains in wind tunnels over a wide Reynolds number range. Desirable is to identify all relevant aeroacoustic sources and to validate the aeroacoustic measurements by comparing them to results of fullscale testings. The aim of the paper is to make a first step for the development of aeroacoustic scaling laws.

\subsection{Methods}

\section{Aerodynamic scaling}

The Reynolds Number is an important non-dimensional parameter in the scope of aerodynamic scaling and can be seen as the ratio of inertia forces and viscous forces:

$$
R e=\frac{c(t) \cdot M a \cdot L \cdot \rho(t)}{\mu(t)} .
$$

Ma denotes the Mach number, $\rho(T)$ the density, $L$ a characteristical length and $\mu(T)$ the dynamic viscosity. Independent of the model's dimensions, for a constant Reynolds number one can expect the same flow topology as long as the shape of the model is the same. This holds as long as compressibility effects are not relevant and the Mach number is small enough $(M a<0.3)$. The Reynolds number is a temperature dependent coefficient, because speed of sound, density and viscosity depend 
on temperature. By cooling down from ambient temperature to $T=100 \mathrm{~K}$ one can increase the Reynolds number by a factor of about 5, at constant Mach number. For more details see [10].

\section{Aeroacoustic "cryo" - scaling}

Temperature variations lead to changes of the physical properties of the fluid which also affect the acoustics. Mainly, acoustics can be influenced in two ways: in amplitude and in observed frequencies. First, some aspects concerning frequency scaling are pointed out below.

As demonstrated later, there are aeroacoustic sources where the frequency is not influenced by the flow velocity within the regarded velocity range at a constant temperature. It seems that the wavelength $\lambda$ is directly connected to a typical length scale or a volume. This behaviour can be described by cavity mode excitation. For this type of sound sources the frequency will be a function of the temperature, because the speed of sound $c$ changes:

$$
f(t)=\frac{c(T)}{\lambda} .
$$

For the comparison of data acquired at different temperatures this effect must be considered. In this particular case it is reasonable to use a dimensionless frequency which is non-dimensionalised by the quotient of the speed of sound and a characteristical length:

$$
f_{\text {norm }}=\frac{f \cdot L}{c}
$$

This formulation is very similar to the well known Strouhal number:

$$
S r=\frac{f \cdot L}{U_{\infty}}=\frac{f \cdot L}{c \cdot M a}
$$

The Strouhal number is a common coefficient to describe another type of sound sources which show a linear dependence between frequency and flow velocity. For instance, in the wake of a cylinder in cross flow one expects a nearly constant Strouhal number over a wide range of Reynolds numbers.

Also the amplitude can be influenced by the temperature. In [12] one can find the derivation for the acoustic source power $P$ of monopole and dipole sound sources, based on the acoustic potential theory. A monopole can be described by a temporal fluctuating source of mass with source strength $\dot{q}$. For a dipole the source strength $\ddot{G}$ has the physical unit force. In a first attempt the assumption is made that the source mechanisms is not a function of the temperature and that there is no impact on the source strength, that means $\dot{q}^{2}(t) \neq F(T)$ and $\ddot{G}^{2}(t) \neq F(T)$ :

$$
\text { Monopole: } \quad P(t)=\frac{\dot{q}^{2}(t)}{4 \pi \rho c} \quad \underset{\dot{q}^{2}(t) \neq F(T)}{\propto} \quad \frac{1}{\rho c}
$$




$$
\text { Dipole: } \quad P(t)=\frac{\ddot{G}^{2}(t)}{12 \pi \rho c^{3}} \quad \underset{\ddot{G}^{2}(t) \neq F(T)}{\propto} \quad \frac{1}{\rho c^{3}}
$$

Then, one obtains a proportionality between source power and viscosity / density which is shown on the right side of the equations. Cooling down from $T=300$ to $T=100$ will lead to a deviation of the sound pressure level less than $\pm 0.25 \mathrm{~dB}$. If experiments show higher deviations, then the source strength must be a function of temperature and/or of the Reynolds number.

\section{Beamforming}

For the sound localisation and quantification in aeroacoustic experiments the Delay and Sum Beamforming algorithm in the frequency domain is applied which is based on the summation of retarded microphone signals. The principles can be find in the textbook from Johnson and Dudgeon [7]. Various modifications of the algorithm were necessary to adopt it to the conditions of measurements in wind tunnels. For more details see $[2,5,16,9,11]$. Beside noise maps, which map the distribution of sound sources, an source integration technique described by Brooks et al. [4] is applied which enables the computation of sound pressure level spectra for specified scan areas.

\subsection{Aeroacoustic measurements}

The 1:25 scaled Inter City Express 3 (ICE3) model is designed for aerodynamic testings and therefore it has a low level of itemisation, which is common for such models. For aerodynamic testings the shape of the model is more important than every single detail. For aeroacoustics this may differ, and single components like antennas, cooling intakes or equipment on the roof are important sources of sound. Nevertheless, the main important details are reproduced, namely the bogies, the pantograph and the gap between head car and first coach. For all considerations concerning the Reynolds number described below, as characteristical length the width $L=0.12 \mathrm{~m}$ of the train is chosen.

\subsubsection{Measurement in the Aeroacoustic Wind tunnel (AWB)}

At first the main aeroacoustic sources are identified by measurements carried out in the Aeroacoustic Wind Tunnel facility (AWB) of the German Aerospace Center

(DLR) in Brunswick [15]. For more details concerning the setup see Lauterbach et al. [11]. The train model was installed on a splitter plate. The aim is to keep the thickness of the boundary layer thin in order to ensure a reasonable simulation of 
the flow underneath the train. In the wind tunnel experiment one expects a Poisseuille flow between splitter plate an train underframe. In the full-scale world the train penetrates the fluid, which is at rest and and a turbulent Couette-like flow will develop. Only a moving belt can provide a better simulation of the full scale world in the wind tunnel, or maybe a mirror model (see Grunwald [6]). However, experiences have shown that the setup on the splitter plate is a good compromise which leads to reasonable results, at least for aerodynamic testings.

Noise maps of the ICE3 can be found in [10]. Here, only a more quantitative representation by focused spectra is given. The three plots in figure 1(a) show spectra, which belong to the areas of the whole train (blue), the pantograph (green) and the first bogie (red). It turns out, that for frequencies higher than $5 \mathrm{kHz}$ the pantograph is the main acoustic source. Further, the pantograph spectrum has strong tonal components. The spectrum of the first bogie also contains tonal components in the low frequency range $<5 \mathrm{kHz}$, but its overall shape is smoother and the sound pressure level declines faster for higher frequencies.

In order to investigate the characteristics of the sound generation at the first bogie and the pantograph, spectra are presented for different flow velocities between $20<U_{\infty}<60 \mathrm{~m} / \mathrm{s}$, corresponding to Reynolds numbers between $0.153 \times 10^{6}<\operatorname{Re}<$ $0.456 \times 10^{6}$. Figure 1 (b) depicts the spectra of the first bogie. The overall shape of the spectra does not change significantly with increasing flow velocity and the two peaks in $f=2455 \mathrm{~Hz}$ and $f=3442 \mathrm{~Hz}$ are not flow velocity depended. Obviously, certain cavity modes are amplified and within the considered velocity range no other modes are excited. Measurements at higher Reynolds numbers are essential to find out if higher acoustic modes can be excited, or whether they persist. At this point it is questionable if it is possible to extrapolate these results to a full scale train.

The pantograph noise differs completely from the bogie noise. Here, a strong velocity dependence of the frequencies is observed. The plot over the Strouhal number points out that there must be a nearly linear frequency - velocity dependence and the spectra show peaks at several constant Strouhal numbers. For more information see Lauterbach et al. [10].

For both, the aerodynamically induced noise from the first bogie and the pantograph a similar power law between observed source power and flow velocity is obtained. For the first bogie one gets $L_{P} \propto U_{\infty}^{6.22}$ and for the pantograph $L_{P} \propto U_{\infty}^{6.12}$.

\subsubsection{Measurement in the Cryogenic Wind tunnel (DNW-KKK)}

The measurements in the aeroacoustic wind tunnel AWB described in the previous section 1.3.1 allow detailed aeroacoustic investigations for Reynolds numbers up to $0.46 \times 10^{6}$. Compared with a full scale train which operates at $R e>16 \times 10^{6}$ this is by a factor of 30 to low and the prediction of the aeroacoustics of a full scale train based on this wind tunnel testings is questionable. Measurements at higher Reynolds numbers are necessary which are presented in this section. As already 
mentioned in the section 1.2 cooling down the fluid is an effective method to increase the Reynolds number. The measurements which are presented in the following have been carried out in the Cryogenic wind tunnel DNW-KKK in Cologne ${ }^{1}$. Figure 2(a) shows a photo of the setup inside the test section. For the same reason as in the AWB, again the model is mounted on a splitter plate. More details concerning the used microphone array can be found in Ahlefeldt et al. [1]. However, the DNWKKK has a high background noise level and the closed test section with hard walls causes a reverberant environment. Additionally, it turns out that the used splitter plate produces more self noise than the splitter plate of the aeroacoustic wind tunnel. Here, all the mountings underneath the plate are exposed to the flow. All these facts lead to a loss of the signal-to-noise ratio (SNR) and limit the measurements. Therefore, in the following third octave spectra are shown.

For a detailed investigation of the influence of the Reynolds number on the aeroacoustic source mechanism of the first bogie, measurements at a constant Mach number of $M a=0.2$ for different temperatures are presented in figure 1(d). Variation of the Reynolds number is realised by varying the temperature of the fluid. Please note that the data are plotted over the non-dimensionalised frequency $f_{\text {norm }}$ (equation 1.3). It turns out that there is no strong influence of the Reynolds number. Within the measuring accuracy the spectra lie on top of each other. The differences in the order of magnitude $\pm 3 \mathrm{~dB}$ can be explained by uncertainties due to the restricted SNR of the measurements under these difficult conditions. There are no clear tendencies of the relation between sound pressure level and Reynolds number.

\subsection{Conclusions \& Outlook}

Two sources of sound with different characteristics were identified by measurements in the AWB. Especially for the noise of the first bogie it is questionable in how far this characteristics persist for higher Reynolds numbers. Therefore measurements at higher Reynolds number were conducted in the DNW-KKK. It turns out that up to $R e=3.6$ mio the aeroacoustic mechanism of the first bogie is similar to what was found in the AWB. A Reynolds number study with constant Mach number shows a weak Reynolds number dependence. Drawback of DNW-KKK is the strong background noise level and reflexions which decrease the SNR. Especially the splitter plate is a strong source of sound and for further investigations a better SNR is desirable. Experiments with a double model are planed (figure 2(b)), which also provides a better simulation of the flow underneath a train. For a moving train this flow can be characterised by a Couette-like flow but in the wind tunnel one will obtain a Poisseuille flow. The flow between the plane of symmetry and the bogie cavity is more comparable to the situation of the moving train.

\footnotetext{
${ }^{1}$ For more information see http://www.dnw.aero.
} 


\section{References}

1. Ahlefeldt, T., Lauterbach, A., Koop, L.: Aeroacoustic measurements of a scaled half model at high reynolds numbers. In: 16th AIAA/CEAS Aeroacoustics Conference, Stockholm, Sweden, AIAA 2010-3748 (2010)

2. Amiet, R.K.: Correction of open jet wind tunnel measurement for shear layer refraction. In: 2nd AIAA Aeroacoustics Conference (AIAA-Paper 75-532, Hampton, USA, 1975)

3. Barsikow, B., King III, W., Pfizenmaier, E.: Wheel/rail noise generated by a high-speed train investigated with a line array of microphones. Journal of Sound and Vibration 118(1),99-122 (1987)

4. Brooks, T., Humphreys, W.: Effect of directional array size on the measurement of airframe noise components. In: 5th AIAA/CEAS Aeroacoustics Conference, Bellevue, USA, AIAAPaper 99-1958 (1999)

5. Ehrenfried, K., Koop, L., Henning, A., Kaepernick, K.: Effects of wind-tunnel noise on array measurements in closed test section. BeBeC-2006-07 (2006). 1st Berlin Beamforming Conference, 22-23 November, 2006

6. Grunwald, K.J.: Aerodynamic characteristics of vehicle bodies at crosswind conditions in ground proximity. Nasa, Langley, Technical Note NASA TN D-5935 (1973)

7. Johnson, D., Dudgeon, D.: Array signal processing: Concepts and techniques. Prentice-Hall, Englewood Cliffs, NJ (1993)

8. King III, W., Pfizenmaier, E., Herrmann, I.: Schallquellen an Hochgeschwindigkeitsstromabnehmern und Moeglichkeiten zur Reduktion. Eine Literaturuebersicht. DFVLR-IB 9251797/B1 (1997). DFVLR, Institut fuer Antriebstechnik, Abteilung Turbulenzforschung, Berlin

9. Lauterbach, A., Ehrenfried, K., Koop, L., Loose, S.: Procedure for the accurate phase calibration of a microphone array. In: 15th AIAA/CEAS Aeroacoustics Conference, Miami, FL, USA, AIAA 2009-3122 (2009)

10. Lauterbach, A., Ehrenfried, K., Koop, L., Loose, S.: Investigation of aeroacoustics of high speed trains in wind tunnels by means of phased microphone array technique. In: Aerodynamics of Heavy Vehicles III: Trucks, Buses and Trains, Potsdam, Germany (2010). Under review

11. Lauterbach, A., Ehrenfried, K., Kroeber, S., Ahlefeldt, T., Loose, S.: Microphone array measurements on high-speed trains in wind tunnels. BeBeC-2010-01 (2010). 3rd Berlin Beamforming Conference, 24-25 February, 2010

12. Lighthill, J.: Waves in Fluids. Cambridge Mathematical Library (1978)

13. Martens, A., Wedemann, J., Meunier, N., Leclere, A.: High speed train noise - sound source localization at fast passing trains. Deutsche Bahn AG, SOCIEDAD ESPAOLA DE ACSTICA, S.E.A. (2009)

14. Pfizenmaier, E., King III, W., Schewe, G., Herrmann, I.: Windkanaluntersuchungen an einem Stromabnehmer fuer den Intercity-Experimental (ICE) der deutschen Bundesbahn. DFVLRIB 22214-85/B5 (1985). DFVLR, Abteilung Turbulenzforschung, Berlin

15. Pott-Pollenske, M., Delfs, J.: Enhanced capabilities of the aeroacoustic wind tunnel Braunschweig. In: 14th AIAA/CEAS Aeroacoustics Conference, Vancouver, British Columbia Canada, AIAA-Paper 2008-2910 (2008)

16. Sijtsma, P.: CLEAN based on spatial source coherence. AIAA Paper 2007-3436 (2007). 13th AIAA/CEAS Aeroacoustics Conference, Rome, Italy, May 21-23, 2007

17. Yamazaki, N., Takaishi, T., Toyooka, M., Nagakura, K., Sagawa, A., Yano, H.: Wind tunnel tests on the control of aeroacoustic noise from high speed train. 9th International Workshop on Railway Noise, Munich (2007) 


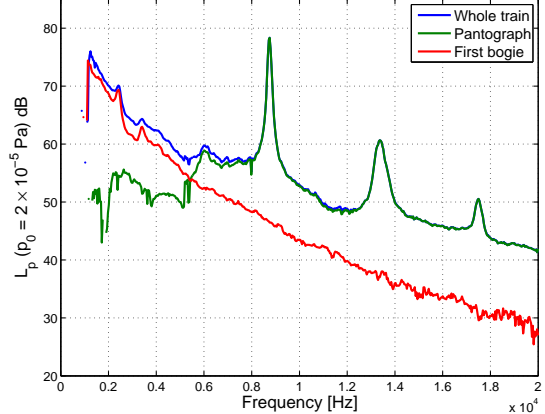

(a) Focused spectra for three different scan areas, at $U_{\infty}=60 \mathrm{~m} / \mathrm{s}$.

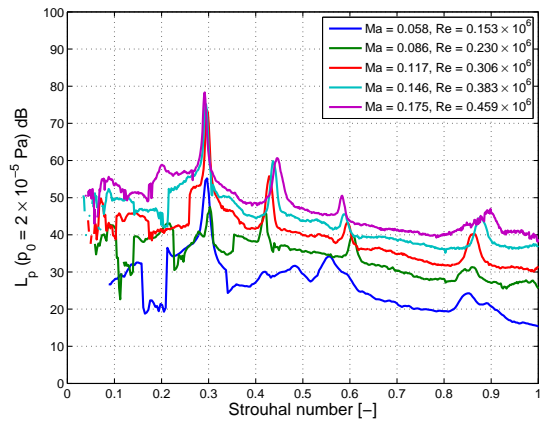

(c) Focused pantograph spectra for $20<U_{\infty}<$ $60 \mathrm{~m} / \mathrm{s}$, plotted over Strouhal number $(L=3$ $\mathrm{mm})$.

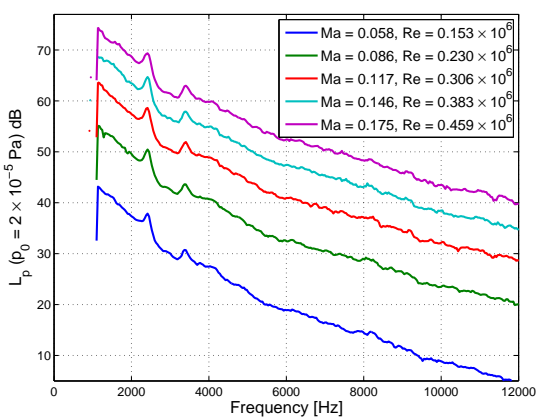

(b) Focused spectra of the first bogie for $20<$ $U_{\infty}<60 \mathrm{~m} / \mathrm{s}$.

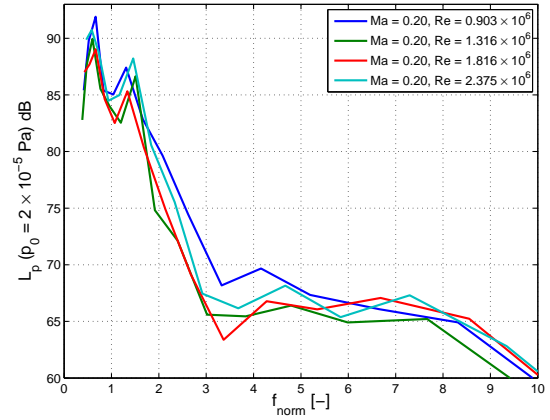

(d) Focused spectra of the first bogie at $M a=$ 0.20

Fig. 1.1 The plots (a) - (c) show spectra acquired in the AWB, figure (d) is based on data measured in the DNW-KKK at $M a=0.2$.

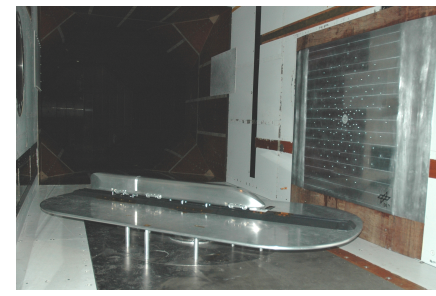

(a) Setup in the test section of the DNW-KKK. The array is mounted on the wind tunnel side wall.

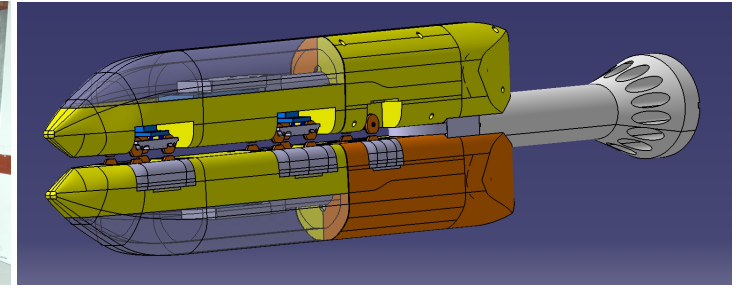

(b) Double model of a train.

Fig. 1.2 Wind tunnel setups and sketch of the planned double model. 